\title{
GENERALIZATION OF REGULARITY AND S-UNITALITY
}

\author{
YONG UK CHO
}

\begin{abstract}
In this paper, we introduce more general concepts of regularity and S-unitality, that is, $\pi$-regularity and $\pi \mathrm{S}$-unitality and then give some examples in near-rings, also investigate their characterization and properties.

AMS Mathematics Subject Classification : 16Y30.

Key words and phrases : regularity, S-unitality, $\pi$-regularity and $\pi \mathrm{S}$ unitality.
\end{abstract}

\section{Introduction}

In 1980, Mason introduced the notions of left regularity, right regularity and strong regularity of near-rings [6, 7]. Moreover, in 1970's and 1986, the concept of $\pi$-regularity was studied by Ligh, Heatherly and Hongan $[2,4,5]$.

The concepts of Von Neumann regularity and $\pi$-regularity in near-ring theory are the same meaning as in ring theory.

Throughout this paper, a near-ring $R$ means a right near-ring [8]. An element $d$ in $R$ is called distributive if $d(a+b)=d a+d b$ for all $a$ and $b$ in $R$.

We will use the following notations: Given a near-ring $R, R_{0}=\{a \in R \mid a 0=$ $0\}$ which is called the zero symmetric part of $R, R_{c}=\{a \in R \mid a 0=a\}$ which is called the constant part of $R$. The set of all distributive elements in $R$ is denoted by $R_{d}$.

Obviously, we see that $R_{0}$ and $R_{c}$ are subnear-rings of $R$, but $R_{d}$ is a semigroup under multiplication. Clearly, near-ring $R$ is zero symmetric, in case $R=R_{0}$ also, in case $R=R_{c}, R$ is called a constant near-ring and in case $R=R_{d}, R$ is called a distributive near-ring.

For notation and basic results, we shall refer to Pilz [8].

Received June 22, 2012. Revised August 3, 2012. Accepted September 3, 2012

(C) 2013 Korean SIGCAM and KSCAM. 


\section{Results}

For a near-ring $R$, an element $a \in R$ is called nilpotent if there exists a positive integer $n$ such that $a^{n}=0$. Also, a subset $S \subset R$ is called nilpotent if there exists a positive integer $n$ such that $S^{n}=0$ and $S \subset R$ is called nil if every element in $S$ is nilpotent, which are introduced in [8]. Clearly, every nilpotent subset of $R$ is nil.

Also, a subset $H$ of $R$ together with (i) $R H \subset H$ and (ii) $H R \subset H$ is called an $R$-subset of $R$. If this $H$ satisfies (i) then it is called a left $R$-subset of $R$, and $H$ satisfies (ii) then it is called a right $R$-subset of $R$.

Also, we say that $R$ is reduced if $R$ has no nonzero nilpotent elements, that is, for each $a$ in $R, a^{n}=0$, for some positive integer $n$ implies $a=0$. McCoy proved that $R$ is reduced iff for each $a$ in $R, a^{2}=0$ implies $a=0$.

A near-ring $R$ is called left $S$-unital (resp. right $S$-unital) if for each $a$ in $R$, $a \in R a$ (resp. $a \in a R$ ), such an element $a$ is called left $S$-unital (resp. right $S$-unital).

$R$ is called $S$-unital, if $R$ is both left S-unital and right S-unital. Every nearring with left identity or identity is clearly left S-unital. Also every regular near-ring is S-unital.

We shall use the phrase " $\forall a \in R, \exists e^{2}=e \in R$ " instead of "for every element $a$ in $R$, there exists some element $e^{2}=e$ in $R$ " for convenience in the following.

Now, we begin with to show the characterization of regularity and S-unitality in near-rings, also consider their application.

Proposition 2.1. Let $R$ be a near-ring. Then $R$ is regular if and only if $R$ has $R a=R e "$ and $R$ is left $S$-unital.

Proof. Suppose that $R$ is regular. Then for any $a \in R$, there exists $x \in R$ such that $a=a x a$. Since $x a$ and $a x$ are idempotents in $R$, taking $x a=e$, $R a=R a x a=R a e \subset R e$ and $R e=R x a \subset R a$. Hence $R a=R e$. Obviously, $R$ is left S-unital.

Conversely, assume that $R$ has the given condition " $\forall a \in R, \exists e^{2}=e \in R$ such that $R a=R e$ " and $R$ is left S-unital. Then S-unitality implies that $a \in R a=R e$, so that there exists $y \in R$ such that $a=y e$. From this condition, we see that $e=e e \in R e=R a$, so that there exists $x \in R$ such that $e=x a$. Thus we obtain that $a=y e=y e e=y e x a=a x a$. Consequently, $R$ is regular.

Corollary 2.2. [1], [3] Let $R$ be a near-ring with identity. Then $R$ is regular if and only if $R$ has the condition " $\forall a \in R, \exists e^{2}=e \in R$ such that $R a=R e$.

The following statements are an application of Proposition 1.

Proposition 2.3. Every regular near-ring $R$ has no non-zero nil left $R$-subset.

Proof. Let $R$ be a regular near-ring and $K$ be a nil left $R$-subset of $R$. It suffices to show that $K=\{0\}$. Indeed, let $a \in K$. Since $R$ is regular, $R$ has the condition $" \exists e^{2}=e \in R$ such that $R a=R e "$ and $R$ is left S-unital, by Proposition 1. Since 
$K$ is a left $R$-subset, we have that $a \in R a \subset K$. On the other hand, since $K$ is nil, there exists positive integer $m$, such that $a^{m}=0$.

Next, from the condition $e=e e \in R e=R a \subset K$, also there exists positive integer $n$, such that $e=e^{n}=0$. From the above two conditions, we have $a \in R 0$, so that $a=r 0$ for some $r \in R$. Consequently, $a=r 0=(r 0)^{m}=a^{m}=0$. That is, $K=\{0\}$.

Corollary 2.4. [1] Every regular near-ring $R$ with identity has no non-zero nil left $R$-subgroup.

From now on, we introduce more general concepts of regularity and S-unitality and then give some examples in near-rings, also investigate their characterization and properties.

Every regular near-ring is $\pi$-regular, but not conversely as following examples.

\section{Example 2.5.}

(1) Let $R=\{0, a, b, c\}$ be an additive Klein 4-group. This is a near-ring with the following multiplication table (p. 408 [8]):

$$
\left(\begin{array}{l|llll}
\cdot & 0 & a & b & c \\
\hline 0 & 0 & 0 & 0 & 0 \\
a & 0 & 0 & a & a \\
b & 0 & a & c & b \\
c & 0 & a & b & c
\end{array}\right)
$$

This near-ring $R$ is a zero-symmetric near-ring with identity $c$. Moreover, $R$ is $\pi$-regular, but not regular. Indeed, $0=0 a 0, a^{2}=a^{2} b a^{2}, b^{4}=b^{4} a b^{4}$, $c^{2}=c^{2} c c^{2}$, but $a$ is not a regular element.

(2) Let $R=\mathbb{Z}_{4}=\{0,1,2,3\}$ be an additive group of integers modulo 4 and define multiplication as follows:

$$
\left(\begin{array}{l|llll}
\cdot & 0 & 1 & 2 & 3 \\
\hline 0 & 0 & 0 & 0 & 0 \\
1 & 0 & 3 & 0 & 1 \\
2 & 0 & 2 & 0 & 2 \\
3 & 0 & 1 & 0 & 3
\end{array}\right)
$$

This near-ring $R$ is a zero-symmetric near-ring without identity. Moreover, $R$ is $\pi$-regular, but not regular. Indeed, $0=0 a 0, a^{2}=a^{2} b a^{2}$, $b^{4}=b^{4} a b^{4}, c^{2}=c^{2} c c^{2}$, but $a$ is not a regular element.

Finally, we can define a general concept of left S-unitality.

A near-ring $R$ is called left $\pi S$-unital (resp. right $\pi S$-unital) if for each $a$ in $R$, there exists a positive integer $n$ such that $a^{n}$ is a S-unital element, that is, $a^{n} \in R a^{n}$ (resp. $a^{n} \in a^{n} R$ ), such an element $a$ is called left $\pi S$-unital (resp. right $\pi S$-unital).

$R$ is called $\pi S$-unital, if $R$ is both left $\pi \mathrm{S}$-unital and right $\pi \mathrm{S}$-unital. 
Also, every left S-unital (resp. right S-unital) near-ring is left $\pi \mathrm{S}$-unital (resp. right $\pi \mathrm{S}$-unital), but not conversely as following remark.

Remark 2.1. In Examples 5 (1), clearly, $R$ is a left S-unital near-ring. But in Examples 5 (1), $R$ is left $\pi \mathrm{S}$-unital, indeed, $0=1 \cdot 0=2 \cdot 0=3 \cdot 0 \in R 0$, $1=3 \cdot 1 \in R 1,2^{2}=0=0 \cdot 2^{2} \in R 2^{2}$ and $3=3 \cdot 3 \in R 3$. But this near-ring $R$ is not S-unital, because 2 is not a left S-unital element.

The statements Proposition 1 and Corollary 2 can be extended on $\pi$-regular and left $\pi \mathrm{S}$-unital near-rings as following.

Theorem 2.6. Let $R$ be a near-ring. Then $R$ is $\pi$-regular if and only if $R$ has the condition " $\forall a \in R, \exists e^{2}=e \in R$ and $\exists n \in Z^{+}$such that $R a^{n}=R e$ ", and $R$ is left $\pi S$-unital.

Proof. Suppose that $R$ is $\pi$-regular. Then for any $a \in R$, there exist a positive integer $n$ and $x \in R$ such that $a^{n}=a^{n} x a^{n}$. This equality implies that $a^{n} \in R a^{n}$. Hence $R$ is left $\pi \mathrm{S}$-unital.

Next, since $x a^{n}$ and $a^{n} x$ are idempotent elements in $R$, putting $x a^{n}=e$, $R a^{n}=R a^{n} x a^{n} \subset R x a^{n}=R e$ and $R e=R x a^{n} \subset R a^{n}$. Hence $R a^{n}=R e$.

Conversely, assume that $R$ has the given condition " $\forall a \in R, \exists e^{2}=e \in R$ and $\exists n \in Z^{+}$such that $R a^{n}=R e^{\prime}$, and $R$ is left $\pi$ S-unital. Then the $\pi$ S-unitality implies that $a^{n} \in R a^{n}=R e$, so that there exists $y \in R$ such that $a^{n}=y e \ldots . .(1)$. On the other hand, we see that $e=e e \in R e=R a^{n}$, so that there exists $x \in R$ such that $e=x a^{n} \ldots . .(2)$. From this two conditions (1) and (2), we obtain that $a^{n}=y e=y e e=y e x a^{n}=a^{n} x a^{n}$. Therefore, $R$ is a $\pi$ S-regular near-ring.

Corollary 2.7. Let $R$ be a near-ring with identity. Then $R$ is $\pi$-regular if and only if $R$ has the condition " $\forall a \in R, \exists e^{2}=e \in R$ and $\exists n \in Z^{+}$such that $R a^{n}=R e^{"}$.

For any near-ring $R$, the center of $R$ is denoted by the set

$$
Z(R)=\{x \in R \mid a x=x a, \forall a \in R\} .
$$

Note that when $R$ is distributive, that is, $R=R_{d}, Z(R)$ is a subnear-ring of $R$. In Appendix of (pp. 421-424 [8]), we can find some distributive $\pi$-regular near-rings which are not additive abelian .

Theorem 2.8. The center of a distributive $\pi$-regular near-ring is also $\pi$-regular.

Proof. Let $R$ be a distributive $\pi$-regular near-ring, and let $a \in Z(R)$. Then $\exists x \in R$ and $\exists n \in Z^{+}$such that $a^{n}=a^{n} x a^{n}$. From this equality, we have that $a^{n}=a^{n} x a^{n}=a^{n} x a^{n} x a^{n}$. We will show that $x a^{n} x \in Z(R)$. Then our claim is done. Indeed, let $t \in R$. Since $a \in Z(R)$, also $a^{n} \in Z(R)$. Thus we can deduce that

$$
t\left(a^{n} x\right)=\left(t a^{n}\right) x=\left(a^{n} t\right) x=a^{n}(t x)=a^{n} x a^{n}(t x)=a^{n} x(t x) a^{n}=a^{n}(x t x) a^{n}
$$

and

$$
\left(a^{n} x\right) t=\left(x a^{n}\right) t=x\left(a^{n} t\right)=x\left(t a^{n}\right)=x t\left(a^{n} x a^{n}\right)=\left(x t a^{n}\right) x a^{n}=a^{n}(x t x) a^{n} .
$$


Hence $a^{n} x \in Z(R)$. Similarly, we can obtain that $x a^{n} \in Z(R)$.

Thus,

and

$$
t\left(x a^{n} x\right)=t\left(a^{n} x x\right)=\left(t a^{n} x\right) x=\left(a^{n} x t\right) x=x\left(a^{n} t\right) x
$$

$$
\left(x a^{n} x\right) t=x\left(a^{n} x\right) t=x t\left(a^{n} x\right)=x\left(t a^{n}\right) x=x\left(a^{n} t\right) x .
$$

This implies that $t\left(x a^{n} x\right)=\left(x a^{n} x\right) t$, that is, $x a^{n} x \in Z(R)$. Hence $Z(R)$ is $\pi$-regular.

Corollary 2.9. The center of a distributive regular near-ring is also regular.

\section{REFERENCES}

1. J.C. Beidleman, A note on regular near-rings, J. Indian Math. Soc. 33 (1969) 207-210.

2. H.E. Heatherly, On regular near-rings, J. Indian Math. Soc. 38 (1974) 345-354.

3. Y. Hirano and H. Tominaga, Regular rings, V-rings and their generalizations, Hiroshima Math. J. 9 (1979) 137-149.

4. M. Hongan, Note on strongly regular near-rings, Proc. Edn Math. Soc. 29 (1986) 379-381.

5. S. Ligh, On regular near-rings, Math. Japon. (1970) 7-13.

6. G. Mason, On strongly regular near-rings, Proc. Edn. Math. Soc. (1980) 27-36.

7. G. Mason, A note on strong forms of regularity for near-rings, Indian J. of Math. (1998) 149-153.

8. G. Pilz, Near-rings, North Holland Publishing Company, Amsterdam, New York, Oxford (1983).

Y. U. Cho received his Ph.D at Kyung Pook National University under the direction of Prof. Young Soo Park in 1987. He visited five universities in U.S.A and Japan as a visiting professor, one time, the University of Louisiana at Lafayette in U.S.A (one year 1996.91997.8), second time, the Ohio University at Athens in U.S.A (one year 2002.3-2003.3), other times, the Okayama University in Japan (1999 one month, 2004 one month, during summer vacations), fifth time, the Florida Atlantic University at Florida in U.S.A (one year 2008.8-2009.7). Now he is acting members of the editorial board of JP-Journal of Algebra (India) from 2000 until now, East Asian Math Journal from 1999 until 2006, and JAMI from 2008 until now. His research interests focus on the theory of near-rings.

Department of Mathematics Education, College of Education, Silla University, Pusan 617736, Korea.

e-mail: yucho@silla.ac.kr 